\title{
Advanced mutation operators applicable in C\# programs
}

\author{
Anna Derezińska \\ Institute of Computer Science, Warsaw University of Technology, Nowowiejska 15/19 \\ 00-665 Warsaw, Poland \\ A.Derezinskalii.pw.edu.pl
}

\begin{abstract}
This paper is devoted to advanced mutation operators for $\mathrm{C \#}$ source code. They deal with object-oriented (OO mutations) and other complex features of the code. They require structural information about a code, unlike the standard mutations. Applicability of $\mathrm{OO}$ operators in $\mathrm{CH}$ is compared with those for other OO languages. Operators for specific features of $\mathrm{C \#}$ language are also proposed. The detailed specification of operators can be provided in terms of pre- and post-conditions of a program transformation. Based on the operators' specification, the generation of mutated $\mathrm{CH}$ programs can be automated.
\end{abstract}

\section{Introduction}

Mutation testing is a fault-based testing technique used for evaluating tests and for measuring the effectiveness of test cases [11]. Mutations are simple changes inserted into a source code. They are defined in terms of mutation operators in order to make the automated testing process. Standard (traditional) mutation operators can be easily specified for many languages, e.g. an operator replacing an arithmetic operator "+" with "-". Testing of new features in object-oriented languages require more complex operators. The changes, introduced by these operators, should be consistent, for instance, with the inheritance hierarchy of classes. These operators take into account information that is non-local to the placement of the change in the source code.

This paper is devoted to advanced mutation operators specialized for $\mathrm{C \#}$ code. They can be more dependent on the programming language than the standard mutation operators. The known (from Java $[3,7,10]$ and $\mathrm{C}++[4]$ ) object-oriented operators were revised and adopted for $\mathrm{C \#}$. Some of the operators have altered definition or different scope of application due to different constructs used in the C\#. New operators for specific, not only object-oriented, features of C\# were also proposed.

Mutation operators were usually defined informally and illustrated by code examples $[3,7,10]$. It is not sufficient for the precise definition of advanced mutation operators. To make a definition unambiguous an operator can be specified as a program transformation with pre- and post-conditions. This approach is presented in the paper. Precise specification of operators allows effectively generating mutated programs (socalled mutants) that could be successively compiled. The specification and the quality of selected operators were verified in experiments on functional and unit tests $[5,6]$.

Please use the following format when citing this chapter: 


\section{Object-oriented mutation testing}

In object-oriented programs standard mutation can be used for intra-method level testing. Object oriented languages provide also new constructions that are not tackled directly by standard mutation operators. The research on the $O O$ mutation was done mostly on Java programs [1,3,7-10]. Mutation of object-oriented features of a UML class specification and $\mathrm{C}++$ code was studied in [4]. To my best knowledge the only research on $\mathrm{OO}$ mutation in C\# was performed by Baudry et al. [2]. They referred to standard mutation operators, invocation of an exception and only two OO operators. The $\mathrm{OO}$ operators were not studied in detail but announced in their Mutator tool. Other C\# mutation tools (Nester) support so far only the standard mutation operators.

An important issue is determining the quality of mutation operators in order to choose the best ones to be applied [8]. A good operator should satisfy the following conditions: (1) reflect typical errors of program developers, (2) generate proper and non-equivalent mutants, (3) be effective in qualification of tests. An equivalent mutant gives for any input exactly the same output as the non-mutated program. The judgment about the equivalence is very effort-consuming. Some mutation operators can generate mutants that are killed very easily by any test. Such operators are not useful in qualification of tests, although they can mimic typical errors of developers. Although $O O$ operators generate fewer mutants than standard operators [9], we would like to limit the number of mutants and choose the most appropriate operators for C\#.

\section{Advanced mutation operators for $\mathrm{C \#}$}

The comprehensive set of mutation operators for $\mathrm{C} \#$ language is presented in tab. 1. The relations for previously defined operators for Java $[3,7,10]$ and/or for $\mathrm{C}++[4]$ are indicated in the column "Ref". The differences between the applicability of the corresponding operators in different languages were examined [5]. Also eight new operators concerning the specific features of $\mathrm{CH}$ were defined. Different groups of operators are discussed below. For the brevity reasons, a description and a specification is given only for two exemplary operators, OPD and IOK.

An informal description of advanced operators is not sufficient for their precise specification. Therefore, any operator could be specified using pre- and postconditions of the transformation of program $P$ to a mutant $P_{\mathrm{O}}^{\prime}$ ( $\mathrm{i}$-th mutant after applying operator $O$ on $P$ ). The pre- and post-conditions are specified using logical predicates with quantifiers (exists $\exists$, for all $\forall$ ) and operators (and, not, or, xor, $\Leftrightarrow, \Rightarrow$ ). In post-conditions, the elements marked with the apostrophe (eg. $\left.x^{\prime}\right)$ relate to elements changed in the mutant $P_{O i^{\prime}}$. Different features of the elements are defined by Boolean values using the dot notation. For example:

x.class True if $x$ is a class

z.override True if $z$ has the modifier override

x.z.method True if $z$ is a method declared in $x$ (or inherited by $x$ ) 
The following expression denotes that $s$ is a syntactically and semantically correct part of instruction $p$ (is used in $p$ ): $s \otimes p$, or equivalently "s" $\otimes p$ for complex $s$. The full notation of the specification and specifications of operators are given in [5].

Table 1. Advanced mutation operators for $\mathrm{CH}$

\begin{tabular}{|c|c|c|c|c|c|}
\hline & Operators & $\operatorname{Inv}$ & Spec & Appl & Ref \\
\hline $\mathrm{AMC}$ & Access modifier change & - & & & {$[10,7,4]$} \\
\hline IHD & Hiding variable deletion & & $=$ & & {$[10,7]$} \\
\hline $\mathrm{IHI}$ & Hiding variable insertion & & - & & {$[10,7]$} \\
\hline IOD & Overriding method deletion & & - & & {$[10,7]$} \\
\hline IOP & Overridden method calling position change & & - & & {$[10,7]$} \\
\hline $\mathrm{IOR}$ & Overridden method rename & - & $\sim$ & - & {$[10]$} \\
\hline ISK & Base keyword deletion & & & & {$[10]$} \\
\hline IPC & Explicit call of a parent's constructor deletion & & - & & {$[10]$} \\
\hline $\mathrm{PNC}$ & New method call with child class type & & - & & {$[10,7]$} \\
\hline PMD & Member variable declaration with parent class type & & & & {$[10,7]$} \\
\hline PPD & Parameter variable declaration with child class type & & & & {$[10,7]$} \\
\hline PRV & Reference assignment with other compatible type & & & & {$[10,4]$} \\
\hline OMR & Overloading method contents change & & & & {$[10]$} \\
\hline OMD & Overloading method deletion & & & - & {$[10,7]$} \\
\hline $\mathrm{OAO}$ & Argument order change & & & - & {$[10,7]$} \\
\hline OAN & Argument number change & & & - & {$[10,7]$} \\
\hline JTD & This keyword deletion & & & & {$[10]$} \\
\hline $\mathrm{JSC}$ & Static modifier change & & & - & {$[10,7]$} \\
\hline JID & Member variable initialization deletion & & & & {$[10]$} \\
\hline JDC & CH-supported default constructor create & & & & {$[10]$} \\
\hline$\overline{\mathrm{EOA}}$ & Reference assignment and content assignment replacement & & & - & {$[10,3]$} \\
\hline EOC & Reference comparison and content comparison replacement & & & - & {$[10,3]$} \\
\hline EAM & Accessor method change & & & - & {$[10,3,4]$} \\
\hline EMM & Modifier method change & & & - & {$[10,3,4]$} \\
\hline MNC & Method name change & & & & {$[3,4]$} \\
\hline MBC & Member changed & & & & [4] \\
\hline $\mathrm{MCO}$ & Member call from another object & & & & [4] \\
\hline $\mathrm{MCI}$ & Member call from another inherited class, MCR in [2] & & & & {$[4,2]$} \\
\hline RFI & Referencing fault insertion & & & & [2] \\
\hline EHR & Exception handler removal & & & & {$[7]$} \\
\hline EHC & Exception handling change & - & & & {$[7]$} \\
\hline$\overline{\mathrm{DMC}}$ & Delegated method change & & & & \\
\hline DMO & Delegated method order change & & & & \\
\hline DEH & Method delegated for event handling change & & & & \\
\hline PRM & Property replacement with member field & & & & \\
\hline $\mathrm{IOK}$ & Override keyword substitution & & & & \\
\hline OPD & Overriding property deletion & & & & \\
\hline OID & Overriding indexer deletion & & & & \\
\hline NDC & Namespace declaration change & & & & \\
\hline
\end{tabular}

Inv - invalid operators (listed for compatibility reasons),

Spec - differences in specification to Java or $\mathrm{C}^{++}$,

Appl - differences in meaning or application scope to Java or C++ 
Several object-oriented inter-class mutation operators can be applied in the similar way in different languages. These operators refer mainly to usage of classes related by inheritance, e. g. PMD, PPD, PRV. Also the operators dealing with incorrect calling of methods are language-independent, e. g. MCO, MNC, MBC, MCI.

Some mutation operators are not appropriate for C\# programs. This was stated by a code analysis and experiments $[5,6]$. These operators (AMC, IOR, EHC) are listed for compatibility reasons and indicated in the column "Inv" of the Table 1.

Other operators for $\mathrm{C \#}$ have to be specified in a different way than the corresponding operators for $\mathrm{C}++$ or Java (the column "Spec" of the table 1). The specification has to take into account new features of $\mathrm{CH}$. For example, extended usage of keywords (new - in operators IHD, IHI), keywords newly introduced in C\# (override - in operators IOD, IOP).

Regardless of an operator specification its application can be different in the considered languages (the column "Appl" in table 1). They can have a different meaning, or the scope of the application can be broader or narrower than that from Java or $\mathrm{C}++$.

The JSC operator for $\mathrm{C \#}$ deletes the static modifier for any member of a class. The reverse operation (adding static modifier as in the JSC operator for Java) could be omitted, because it provides non-compiled code in most cases.

The EOA operator replaces assignment of an object reference pointing to an object with the clone (duplicate) of this object. It intends to check a possible mismatch of objects and object references. In C\# the overloaded Clone method creates an object duplicate and is defined for many types. The EOA operator can be applied for a class which has its Clone() method.

The EOC operator replaces one kind of comparison with another one (= with EqualsO or v.v.). In C\# the default Object.Equals method calls Object.ReferenceEquals which results in a reference comparison instead of a value comparison. For many types Equals method is overloaded to implement value comparison. The user can overload Equals method and the operator $=$ for own declared types.

Operators EAM and EMM dealing with accessor and modifier methods (get and set) have a minor significance in $\mathrm{C \#}$ because a new element - property can be used.

New mutation operators for the specific features of $\mathrm{C} \#$ were also defined. They are dealing with delegates, properties, indexers, override modifier and namespaces. Properties are values that can be stored or retrieved of a class using an accessor (get) and a modifier (set). Properties can be overriding in the similar way as methods do. The OPD operator deletes a whole definition of a property from the derived class, e. g.:

\section{OPD: Original code}

public class Figure

public virtual double Area

\{

\}

get return $0 ;\}$

\}

public class Square : Figure

$\{$ public override double Area

$\{$ get

\}
Mutated code

public class Figure

\{ public virtual double Area

$\{$ get

\}

\{ return $0 ;\}$

\}

public class Square : Figure

\{ return Math.Ar(base. 2); \} 
The operator forces the usage of the appropriate property from the base class. It can be applicable only if the class does not inherit from an abstract type, otherwise a compilation error would be detected because the class does not implement inherited abstract member. A specification of the OPD operator is given below:

\section{OPD Pre: $\quad \exists_{x} x$.class and $\exists_{y} y$.class and $y . x$.public inherited and and not $x$.abstract and $\exists_{z}$ (y.z.property and z.override) \\ OPD Post: $\quad$ not $y^{\prime} \cdot z^{\prime}$.property}

Indexers are used to index a class in the same way as an array. The OID operator deletes a whole definition of an indexer from the derived class. This operator can be defined in the similar way as the OPD operator.

In properties the set modifier uses an implicit parameter called value, whose type is of the property. By convention names of properties begin with capital letter, while names of fields with a small one. By mistake a property can be called instead of a field or vice versa. The PRM operator replaces those two names.

Omission of the keyword override in a method declaration is a common mistake of $\mathrm{C} \#$ program developers, who have habits from $\mathrm{C}++$ or Java. In C\#, special keywords (override, new) denote override or hide of a method from the base class. The IOK operator substitutes an override occurrence with the new keyword, or vice versa (new with override). This substitution cannot be revealed by a compiler.

\section{IOK: Original code}

public class Figure

$\{$ public virtual void Draw()

\}

$$
\{\ldots .\}
$$

public class Square : Figure

$\{$ public override void Draw()

\}

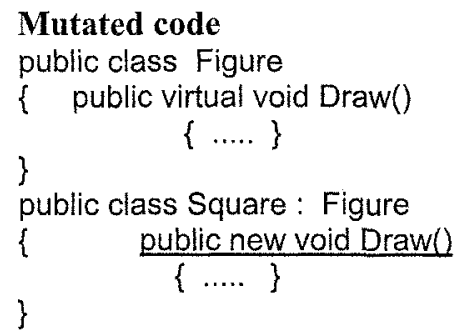

Mutated code public class Figure

$\{$ public virtual void Draw()

\}

public class Square : Figure

\}

This mutation will be detected if polymorphism is used. In the above example an object of Square can be referenced as a Figure. After mutation a method Drow called for this object will invoke a method from class Figure instead of class Square.

IOK Pre:

$\exists_{\mathrm{x}} \mathrm{x}$.class and $\exists_{\mathrm{y}} \mathrm{y}$.class and y.x.public_inherited and

$\exists_{z}$ (x.z.method and (z.override or z.new))

IOK Post: $\quad$ (z.override $\Rightarrow z^{\prime}$.new) and (z.new $\Rightarrow z^{\prime}$.override)

In $\mathrm{C} \#$ delegates are the object-oriented equivalents of function pointers. However, unlike function pointers, delegates are type-safe and secure. Delegates can be used in callback and event-handling scenarios. The DMC operator changes a delegated method into another method visible in this context and taking the same types of parameters. The operator simulates a fault, when a developer used by mistake a different method as a callback. The DMO operator changes the order of assignment of delegated methods. The DEH operator changes a method delegated for the event handling. Simulated fault can be for example caused by misleading of elements during construction of a GUI. The operators on delegates are extensively studied in [6].

The namespace statement is used in C\# to define a new namespace, which encapsulates the classes. The NDC operator changes a namespace declaration. It is used only if exists an appropriate declaration of the class in both namespaces. 


\section{Final Remarks}

The object-oriented mutation operators adopted for $\mathrm{CH}$ programs and other advanced operators dealing with new programming features were studied. Defining a mutation operator as a program transformation with pre- and post-conditions allows to give a precise specification of the operator. It is especially important for complex operators dealing with structural features of a program. Based on provided specifications of operators a tool for mutation of $\mathrm{C} \#$ programs is currently under development.

The application of selected mutation operators for $\mathrm{C} \#$ was evaluated in experiments. They allowed verifying the specifications, comparing usefulness of operators and suitability for the test selection. The sets of functional tests and unit tests were used $[5,6]$. The preliminary results showed that object-oriented operators IHD, IHI, IOD, IOP, IOK, OPD and OMD generated proper, non-equivalent mutants and were selective in assessment of the quality of functional tests. Mutants generated by the PRM operator were non-equivalent but killed by all functional tests. Among the operators dealing with exception handling and delegates two operators EHR and DMC were the most promising ones. The evaluation of mutation operators for $\mathrm{C \#}$ and comparison with other testing criteria needs still further experiments.

Acknowledgment This work was supported by the Polish State Committee for Scientific Research under the project 4 T 11 C 04925.

\section{References}

1. Alexander, R. T., Bieman, J. M., Ghosh, J. M., Bixia, J.: Mutation of Java objects, Proc of $13^{\text {th }}$ Int. Symp. on Software Reliability Eng., (2002) 341-351

2. Baudry, B., Fleurey, F., Jezequel, J-M., Traon, Y. Le.: From genetic to bacteriological algorithms for mutation-based testing, Sof. Testing Verif. and Reliab., vol 15, no 2, (2005)

3. Chevalley, P.: Applying mutation analysis for object-oriented programs using a reflective approach, Proc of 8-th Asia-Pacific Softw. Engin. Conf., ASPEC (2001) 267-270

4. Derezińska, A.: Object-oriented mutation to assess the quality of tests, Proc. of $29^{\text {th }}$ Euromicro Conf., Belek, Turkey, 1-6 Sept. 2003, IEEE Comp. Soc. (2003) 417-420

5. Derezińska, A.: Specification of mutation operators specialized for C\# code, ICS Res. Raport 2/05 WUT (2005)

6. Derezińska, A.: Quality assessment of mutation operators dedicated for C\# programs, accepted for Inter. Conf. on Quality Software, QSIC06, Beijing, China, Oct. (2006)

7. Kim, S., Clark, J., McDermid J. A.: Class Mutation: mutation testing for object-oriented programs, Proc of Conf, on Object-Oriented Soft. Systems, Erfurt, Germany, Oct. (2000)

8. Kim, S, Clark, J., McDermid J. A.: Investigating the effectiveness of $O O$ testing strategies with the mutation method, J. of Soft. Testing, Verif, and Rel., 11(4) (2001) 207-225

9. Ma, Y-S., Offutt, J., Kwon, Y-R.: MuJava: an automated class mutation system, Softw, Testing, Verif. and Reliab., vol 15, no 2, June (2005)

10. Ma, Y-S., Kwon, Y-R., Offutt, J.: Inter-class mutation operators for Java, Proc. of Inter. Symp. on Software Reliability Engin., ISSRE'02, IEEE Computer Soc., (2002)

11. Voas, J.M., McGraw, G.: Software fault injection, Inoculating programs against errors, John Wiley \& sons Inc. (1998) 